\begin{tabular}{c|c|c|} 
SELECCIONES MATEMÁTICAS \\
Universidad Nacional de Trujillo \\
ISSN: $2411-1783$ (Online) \\
2020; Vol. 7(2): 234-241.
\end{tabular}

\title{
Equivalence of the stability of discrete-time Markov jump linear systems
}

\section{Jorge Enrique Mayta Guillermo® and Maritza Lourdes Moreno Capristano®}

Received, Mar. 18, 2020

Accepted, Oct. 13, 2020

How to cite this article:

Mayta Guillermo JE, Moreno Capristano ML. Equivalence of the stability of discrete-time Markov jump linear systems. Selecciones Matemáticas. 2020;7(2):234-241. http://dx.doi.org/10.17268/sel.mat.2020. 02.05

\begin{abstract}
This paper investigates the stability of discrete-time Markov jump linear system of second-order, this type of system is similar to the family of discrete-time Markov jump linear system it is known in classical literature as MJLS. We present some consistent stability definitions for the system, where these types of stability are equivalent as long as the state space Markov chain is finite. In addition, a computational test is presented to analyze the stability of the system. The result is a generalization of classical theory, this implies a contribution to the theory.
\end{abstract}

Keywords . Stability, discrete-time Markov jump linear system, Markov chain.

1. Introduction. The Markov jump linear system is a special class of stochastic control systems with multi-mode. The main problems in control systems is their ability to maintain stable behavior and meet some performance requirements even in the presence of abrupt changes in system dynamics. These changes may be due, for example, to abrupt environmental disturbances, component failures or repairs, changes in subsystem interconnections, abrupt changes in the operating point for a plant. These systems are presented in the control of solar thermal central receivers, aircraft control systems, economic systems, robotic handling systems, etc. In addition the applications are found in power systems [1], [2], satellite control [3], flight control [4], air traffic mgmt [5], solar thermal receiver [6], cell growth [7], etc.

Several authors have studied this system and, in particular due to some applications, which have aroused great interest in this subject, the literature has constantly increased for example [8, 9, 10]. Analyzing the stability of the system is a very important problem, it can be found in $[11,12,13,14]$.

The paper is organized as follows: In the section 2 gives the basic notations that will be used in the article. In the section 3, homogeneous linear systems with second-order Markovian jumps will be presented, which will impose certain conditions for its stability analysis. In the section 4 it consists of three subsections. In the first part some stability definitions for this system will be announced, a matrix will be presented which stores the probabilistic information of the Markov chain and the values of the matrices associated with the system. In the second part the stability is analyzed by means of the spectral radius of the matrix.In the third part the equivalence between the types of stability defined in section five will be presented. Finally we will give some comments and culminate with the conclusions.

2. Basic Notation. In this section, the general notation used throughout the paper and certain specifications of the problem to be addressed are given. In this section, we will introduce the basic notation we use in all the work. The set of natural numbers is denoted by $\mathbb{N}$ and the $n$-dimensional euclidean space of real

*Facultad de Ciencias, Universidad Nacional de Ingeniería, Perú (jmaytag@uni .edu •pe).

${ }^{\dagger}$ Facultad de Ciencias, Universidad Nacional de Ingeniería, Perú(mmorenoc@uni . edu • pe). 
numbers is denoted by $\mathbb{R}^{n}$, the euclidean norm is denoted by $\|\cdot\|$, the set of non-negative integer numbers, by $\mathbb{Z}_{+}$. The space of matrices of order $m \times n$ on $\mathbb{R}$ is denoted by $\mathbb{R}^{m \times n}$. The transpose matrix and the inverse of the transpose matrix of $A$ is denoted by $A^{T}$ and $A^{-T}$, respectively and the identity matrix of order $n \times n$ is denoted by $I_{n}$.

The spectral radius of $A \in \mathbb{R}^{n \times n}$ is denoted by $\rho(A)$. The Kronecker product is denoted with the symbol $\otimes$ and the stacking column vector operator by $\operatorname{vec}(\cdot)$.

Given that the work we do in this article is in the probabilistic sense it is necessary to define a probability space $(\Omega, \mathcal{F}, \mathbb{P})$, where $\Omega$ is the sample space, $\mathcal{F}$ is the sigma algebra of events and $\mathbb{P}$ is the probability measure, which are defined random variables.

A state random variable is written in boldface and its expectation is denoted by $\mathbb{E}\{\cdot\}$. A discrete-time process is denoted by $\{\boldsymbol{x}(k)=\boldsymbol{x}(k, w)\}_{k \in \mathbb{Z}_{+}}$. The indicator function with respect to $A \in \mathcal{F}$, is denoted by $1_{A}$. The Markov Chain $\{\boldsymbol{\theta}(k)\}_{k \in \mathbb{Z}_{+}}$takes values in $\mathcal{L} \triangleq\{1, \ldots, L\}, L \in \mathbb{N}$. The Markov Chain $\{\boldsymbol{\theta}(k)\}_{k \in \mathbb{Z}_{+}}$is taken to be homogeneous with transition probability matrix $\Pi=\left[p_{i j}\right]_{L \times L}$, where

$$
p_{i j}=\mathbb{P}\{\boldsymbol{\theta}(k+1)=j \mid \boldsymbol{\theta}(k)=i\},
$$

and initial state probability vector $\pi=\left(\pi_{1}, \cdots, \pi_{L}\right)$, where $\pi_{i}=\mathbb{P}(\boldsymbol{\theta}(0)=i)$.

3. Problem Formulation. The System

$$
\boldsymbol{x}(k+2)=A_{\boldsymbol{\theta}(k+1)} \boldsymbol{x}(k+1)+B_{\boldsymbol{\theta}(k)} \boldsymbol{x}(k),
$$

where for all $i, j \in \mathcal{L}, A_{i}, B_{j} \in \mathbb{R}^{n \times n}, \boldsymbol{x}(k) \in \mathbb{R}^{n \times 1}$. We will assume that the initial conditions $\{\boldsymbol{x}(0), \boldsymbol{x}(1)\}$ y $\{\boldsymbol{\theta}(0), \boldsymbol{\theta}(1)\}$ they are independent, also $\boldsymbol{x}(0), \boldsymbol{x}(1)$ they are second moment finite, i.e., $\mathbb{E}\left\{\|\boldsymbol{x}(0)\|^{2}\right\}<\infty$ and $\mathbb{E}\left\{\|\boldsymbol{x}(1)\|^{2}\right\}<\infty$. Here is the definition of system solution of (3.1).

Definition 3.1. It is said that the stochastic process $\boldsymbol{x}(k)=\{\boldsymbol{x}(k)\}_{k \in \mathbb{Z}_{+}}$is solution of (3.1) if for any realization $\omega$ of $\theta(k)$ and for any $\boldsymbol{x}(0)$ and $\boldsymbol{x}(1)$, the Equation (3.1) is satisfied point wise, that is,

$$
\boldsymbol{x}(k+2, \omega ; \boldsymbol{x}(0), \boldsymbol{x}(1))=A_{\boldsymbol{\theta}(k+1, \omega)} \boldsymbol{x}(k+1, \omega ; \boldsymbol{x}(0), \boldsymbol{x}(1))+B_{\boldsymbol{\theta}(k, \omega)} \boldsymbol{x}(k, \omega ; \boldsymbol{x}(0), \boldsymbol{x}(1)), k \in \mathbb{Z}_{+} .
$$

The solution $\boldsymbol{x}(k)$ of the System (3.1) it is also called trajectory solution.

Now, making the change of variable

$$
\boldsymbol{z}(k)=\left[\begin{array}{c}
\boldsymbol{x}(k) \\
\boldsymbol{x}(k+1)
\end{array}\right]
$$

the System (3.1) it can be expressed as follows

$$
\boldsymbol{z}(k+1)=C_{\hat{\boldsymbol{\theta}}(k)} \boldsymbol{z}(k), \boldsymbol{z}(0) \in \mathbb{R}^{2 n \times 1},
$$

where $\boldsymbol{z}(k) \in \mathbb{R}^{2 n \times 1}, \hat{\boldsymbol{\theta}}(k)=(\boldsymbol{\theta}(k), \boldsymbol{\theta}(k+1))$ and

$$
C_{\hat{\boldsymbol{\theta}}(k)}=\left[\begin{array}{cc}
0 & I_{n} \\
B_{\boldsymbol{\theta}(k)} & A_{\boldsymbol{\theta}(k+1)}
\end{array}\right] .
$$

In addition, the following equality and inequality is obtained

$$
\|\boldsymbol{z}(k)\|^{2}=\|\boldsymbol{x}(k)\|^{2}+\|\boldsymbol{x}(k+1)\|^{2} \text { and }\|\boldsymbol{x}(k)\| \leq\|z(k)\| .
$$

We observe that the System (3.2) it is similar to a MJLS, what would be missing to verify that $\hat{\boldsymbol{\theta}}(k)$ is a Markov chain.

Lemma 3.1. The $\boldsymbol{\theta}(k)$ a Markov chain with state space $\mathcal{L}$. Then the stochastic process $\hat{\boldsymbol{\theta}}(k)$ defined by

$$
\hat{\boldsymbol{\theta}}(k)=(\boldsymbol{\theta}(k), \boldsymbol{\theta}(k+1)),
$$

is an irreducible Markov chain with state space

$$
\mathcal{L} \times \mathcal{L}=\{(i, j) \mid i, j \in \mathcal{L}\},
$$

and the probability of transitions as follows:

$$
\mathbb{P}\{\hat{\boldsymbol{\theta}}(k+1)=(s, r)) \mid \hat{\boldsymbol{\theta}}(k)=(i, j)\}=\left\{\begin{array}{cc}
0 & \text {, if } j \neq s \\
p_{j r} & , \text { if } j=s
\end{array} .\right.
$$


Proof: We notice that the states space of $\hat{\boldsymbol{\theta}}(k)$ is $\mathcal{L} \times \mathcal{L}$. We will verify the Markovian property, in fact for this let's take $\hat{i}_{0}, \hat{i}_{1} \ldots \hat{i}_{k+1}$ arbitrarily in $\mathcal{L} \times \mathcal{L}$ it follows:

$\mathbb{P}\left\{\hat{\boldsymbol{\theta}}(k+1)=\hat{i}_{k+1} \mid \hat{\boldsymbol{\theta}}(k)=\hat{i}_{k} \ldots \hat{\boldsymbol{\theta}}(0)=\hat{i}_{0}\right\}=\frac{\mathbb{P}\left\{\boldsymbol{\theta}(k+2)=r, \boldsymbol{\theta}(k+1)=s, \boldsymbol{\theta}(k+1)=j, \ldots \boldsymbol{\theta}(0)=i_{0}\right\}}{\mathbb{P}\left\{\boldsymbol{\theta}(k+1)=j, \ldots \boldsymbol{\theta}(0)=i_{0}\right\}}$

Analyzing by cases, if $j \neq s$ then

$$
\mathbb{P}\left\{\hat{\boldsymbol{\theta}}(k+1)=\hat{i}_{k+1} \mid \hat{\boldsymbol{\theta}}(k)=\hat{i}_{k} \ldots \hat{\boldsymbol{\theta}}(0)=\hat{i}_{0}\right\}=0,
$$

on the other hand

$$
\mathbb{P}\left\{\hat{\boldsymbol{\theta}}(k+1)=\hat{i}_{k+1} \mid \hat{\boldsymbol{\theta}}(k)=\hat{i}_{k}\right\}=0
$$

Now, if $j=s$

$\mathbb{P}\left\{\hat{\boldsymbol{\theta}}(k+1)=\hat{i}_{k+1} \mid \hat{\boldsymbol{\theta}}(k)=\hat{i}_{k} \ldots \hat{\boldsymbol{\theta}}(0)=\hat{i}_{0}\right\}=\frac{\mathbb{P}\left\{\boldsymbol{\theta}(k+2)=r, \boldsymbol{\theta}(k+1)=j, \boldsymbol{\theta}(k+1)=j, \ldots \boldsymbol{\theta}(0)=i_{0}\right\}}{\mathbb{P}\left\{\boldsymbol{\theta}(k+1)=j, \ldots \boldsymbol{\theta}(0)=i_{0}\right\}}=p_{j, r}$,

on the other hand

$$
\mathbb{P}\left\{\hat{\boldsymbol{\theta}}(k+1)=\hat{i}_{k+1} \mid \hat{\boldsymbol{\theta}}(k)=\hat{i}_{k}\right\}=\frac{\mathbb{P}\{\boldsymbol{\theta}(k+2)=r, \boldsymbol{\theta}(k+1)=j, \boldsymbol{\theta}(k)=i\}}{\mathbb{P}\{\boldsymbol{\theta}(k+1)=j, \boldsymbol{\theta}(k)=i\}}=p_{j, r} .
$$

This proves the Markovian property of $\hat{\theta}(k)$.

4. Stability. This section presents some stability definitions for the System (3.1), which are adaptations of the theory of MJLS (see [15]).

Definition 4.1. The System (3.1) is:

a) MSS (Mean square stable) if for any $\boldsymbol{\theta}(0), \boldsymbol{\theta}(1)$ and any initial condition $\boldsymbol{x}(0), \boldsymbol{x}(1)$,

$$
\lim _{k \rightarrow \infty} \mathbb{E}\left\{\boldsymbol{x}(k) \boldsymbol{x}^{T}(k)\right\}=0 .
$$

b) SS (Stochastically stable) if for any $\boldsymbol{\theta}(0), \boldsymbol{\theta}(1)$ and any initial condition $\boldsymbol{x}(0), \boldsymbol{x}(1)$,

$$
\sum_{k=0}^{\infty} \mathbb{E}\left\{\|\boldsymbol{x}(k)\|^{2}\right\}<\infty
$$

c) MSES (Mean square Exponentially Stable) if for any $\boldsymbol{\theta}(0), \boldsymbol{\theta}(1)$ and any initial condition $\boldsymbol{x}(0), \boldsymbol{x}(1)$ there exist constants $1 \leq \beta$ and $0<\gamma<1$ and such that for all $k \geq 0$,

$$
\mathbb{E}\left\{\|\boldsymbol{x}(k)\|^{2}\right\} \leq \beta \gamma^{k} \mathbb{E}\left\{\|\boldsymbol{x}(0)\|^{2}\right\}
$$

d) ASS (Almost sure stable) if for any $\boldsymbol{\theta}(0), \boldsymbol{\theta}(1)$ and any initial condition $\boldsymbol{x}(0), \boldsymbol{x}(1)$,

$$
\mathbb{P}\left\{\lim _{k \rightarrow \infty}\|\boldsymbol{x}(k)\|=0\right\}=1
$$

In this section the matrix is presented $\mathcal{A}$ which will be very useful to analyze the stability of the System (3.1). Therefore, it is expected that system stability can be analyzed through the spectral radius of this matrix as classical theory. In order to introduce these matrices we need to define some matrices that are given in terms of the solution path of the system. These matrices, which will be used throughout this work, are consistent with those defined in the in classical literature. 
For each $k \in \mathbb{Z}_{+},(i, j) \in \mathcal{L} \times \mathcal{L}$, let's consider

$$
\begin{aligned}
\hat{Q}(k) & =\mathbb{E}\left\{\boldsymbol{z}(k) \boldsymbol{z}^{T}(k)\right\}, \\
\hat{Q}_{i, j}(k) & =\mathbb{E}\left\{\boldsymbol{z}(k) \boldsymbol{z}^{T}(k) 1_{\{\hat{\boldsymbol{\theta}}(k)=(i, j)\}}\right\}, \\
\hat{q}_{i, j}(k) & =\operatorname{vec}\left(\hat{Q}_{i, j}(k)\right), \\
\hat{q}(k) & =\left(\begin{array}{c}
\hat{q}_{1,1}(k) \\
\vdots \\
\hat{q}_{1, L}(k) \\
\vdots \\
\hat{q}_{L, 1}(k) \\
\vdots \\
\hat{q}_{L, L}(k)
\end{array}\right)
\end{aligned}
$$

Note that

$$
\begin{aligned}
\hat{Q}(k) & =\mathbb{E}\left\{\sum_{(i, j) \in \mathcal{L} \times \mathcal{L}} \boldsymbol{z}(k) \boldsymbol{z}^{T}(k) 1_{\{\hat{\boldsymbol{\theta}}(k)=(i, j)\}}\right\} \\
& =\sum_{(i, j) \in \mathcal{L} \times \mathcal{L}} \hat{Q}_{i, j}(k) .
\end{aligned}
$$

The following relationships are very well known results that are going to be useful in our derivations [?].

$$
\frac{1}{n} \mathbb{E}\left\{\|\boldsymbol{x}(k)\|^{2}\right\} \leq\left\|\mathbb{E}\left\{\boldsymbol{x}(k) \boldsymbol{x}^{T}(k)\right\}\right\| \leq \mathbb{E}\left\{\|\boldsymbol{x}(k)\|^{2}\right\}, \boldsymbol{x}(k) \in \mathbb{R}^{n \times 1} .
$$

The Lemma 4.1 establish a recursive equation for the matrix $\hat{Q}_{i, j}(k)$, defined in (4.2). This equation is very useful for obtaining the main results presented in this work.

Lemma 4.1. The System (3.2) with solution $z(k)$, the matrix $\hat{Q}_{i, j}(k)$ defined in (4.2) satisfy the following recursive equation:

$$
\hat{Q}_{j, r}(k+1)=\sum_{i=1}^{L} p_{j, r} C_{i, j} \hat{Q}_{i, j}(k) C_{i, j}^{T} i, j \in \mathcal{L}, k \in \mathbb{Z}_{+} .
$$

Proof: For the proof of this result we use the recursion of the System (3.2).

$$
\begin{aligned}
\hat{Q}_{j, r}(k+1) & =\mathbb{E}\left\{\boldsymbol{z}(k+1) \boldsymbol{z}^{T}(k+1) 1_{\{\hat{\boldsymbol{\theta}}(k+1)=(j, r)\}}\right\} \\
& =\mathbb{E}\left\{C_{\hat{\boldsymbol{\theta}}(k)} \boldsymbol{z}(k) \boldsymbol{z}^{T}(k) C_{\hat{\boldsymbol{\theta}}(k)}^{T} 1_{\{\hat{\boldsymbol{\theta}}(k+1)=(j, r)\}}\right\} \\
& =\mathbb{E}\left\{\sum_{i=1}^{L} C_{i, j} \boldsymbol{z}(k) \boldsymbol{z}^{T}(k) C_{i, j}^{T} 1_{\{\boldsymbol{\theta}(k)=i, \hat{\boldsymbol{\theta}}(k+1)=(j, r)\}}\right\} \\
& =\sum_{i=1}^{L} C_{i, j} \mathbb{E}\left\{\boldsymbol{z}(k) \boldsymbol{z}^{T}(k) 1_{\{\boldsymbol{\theta}(k)=i, \hat{\boldsymbol{\theta}}(k+1)=(j, r)\}}\right\} C_{i, j}^{T} \\
& =\sum_{i=1}^{L} C_{i, j} \mathbb{E}\left\{\mathbb{E}\left\{\boldsymbol{z}(k) \boldsymbol{z}^{T}(k) 1_{\{\boldsymbol{\theta}(k)=i, \hat{\boldsymbol{\theta}}(k+1)=(j, r)\}} \mid \boldsymbol{z}(k), \hat{\boldsymbol{\theta}}(k)\right\}\right\} C_{i, j}^{T} \\
& =\sum_{i=1}^{L} C_{i, j} \mathbb{E}\left\{\boldsymbol{z}(k) \boldsymbol{z}^{T}(k) 1_{\{\hat{\boldsymbol{\theta}}(k)=(i, j)\}} \mathbb{E}\left\{1_{\{\boldsymbol{\theta}(k+2)=r\}} \mid \boldsymbol{z}(k), \hat{\boldsymbol{\theta}}(k)\right\}\right\} C_{i, j}^{T} \\
& =\sum_{i=1}^{L} C_{i, j} \mathbb{E}\left\{\boldsymbol{z}(k) \boldsymbol{z}^{T}(k) 1_{\{\hat{\boldsymbol{\theta}}(k)=(i, j)\}} p_{j, r}\right\} C_{i, j}^{T} \\
& =\sum_{i=1}^{L} p_{j, r} C_{i, j} \hat{Q}_{i, j}(k) C_{i, j}^{T} .
\end{aligned}
$$


The matrix $\mathcal{A}$ is defined below which will be of fundamental importance to establish different results related to the stability of the System (3.1) and the System (3.2).

$$
\mathcal{A} \triangleq\left(\bar{\Pi}^{T} \otimes I_{4 n^{2}}\right) \operatorname{diag}\left[C_{i, j} \otimes C_{i, j}\right]
$$

where the matrix $\operatorname{diag}\left[C_{i, j} \otimes C_{i, j}\right]$ it is a diagonal matrix by blocks and $\bar{\Pi}$ is the probability transition matrix of the Markov chain $\hat{\theta}(k)$ which is as follows:

$$
\bar{\Pi}=D \cdot\left(I_{L} \otimes \Pi\right),
$$

where

$$
D=\left[\begin{array}{ccc}
D_{1} & \ldots & D_{L} \\
\vdots & \ddots & \vdots \\
D_{1} & \ldots & D_{L}
\end{array}\right]
$$

and

$$
\mathcal{D}_{i}=\left[\begin{array}{ccccc}
0 & \cdots & 0 & \cdots & 0 \\
\vdots & \ddots & \vdots & \ddots & \vdots \\
0 & \cdots & 1 & \cdots & 0 \\
\vdots & \ddots & \vdots & \ddots & \vdots \\
0 & \cdots & 0 & \cdots & 0
\end{array}\right]_{L \times L} \rightarrow \text { i-row }
$$

$$
\uparrow \text { i-column }
$$

For better block matrix operation $\operatorname{diag}\left[C_{i, j} \otimes C_{i, j}\right]$, present a particular case, for case $L=2$ is obtained

$$
\operatorname{diag}\left[C_{i, j} \otimes C_{i, j}\right]=\left[\begin{array}{cccc}
C_{1,1} \otimes C_{1,1} & 0 & 0 & 0 \\
0 & C_{1,2} \otimes C_{1,2} & 0 & 0 \\
0 & 0 & C_{2,1} \otimes C_{2,1} & 0 \\
0 & 0 & 0 & C_{2,2} \otimes C_{2,2}
\end{array}\right]
$$

Note that these matrices collect the information of all the system parameters and also keep the probable information of the Markov chain. Next it shows that the System (3.2) it can be transformed into an equation of the classical type by the matrix $\mathcal{A}$.

Lemma 4.2. The column vector $\hat{q}(k)$ defined in (4.4), is the solution of

$$
y(k+1)=\mathcal{A} y(k), y(0)=\hat{q}(0) \in \mathbb{R}^{4 n^{2}} .
$$

Proof:

Vectorizing both sides of (4.7) is obtained

$$
\hat{q}_{j, r}(k+1)=\sum_{i=1}^{L} p_{j, r} C_{i, j} \otimes C_{i, j} \hat{q}_{i, j}(k),
$$

what can be written matrixally in the following way:

$$
\hat{q}(k+1)=\mathcal{A} \hat{q}(k),
$$

and by induction you have

$$
\hat{q}(k)=\mathcal{A}^{k} \hat{q}(0) .
$$


Lemma 4.3. If $\lim _{k \rightarrow \infty} \mathbb{E}\left\{\boldsymbol{z}(k) \boldsymbol{z}^{T}(k)\right\}=0$ if and only if for any $\boldsymbol{x}(0), \boldsymbol{x}(1) \in \mathbb{R}^{n}$ and for any $\boldsymbol{\theta}(0), \boldsymbol{\theta}(1)$ is fulfilled

$$
\lim _{k \rightarrow+\infty} \hat{q}(k)=0 .
$$

Proof: Let's assume that $\lim _{k \rightarrow \infty} \mathbb{E}\left\{\boldsymbol{z}(k) \boldsymbol{z}^{T}(k)\right\}=0$. The inequality

$$
\begin{aligned}
\left\|\hat{Q}_{i, j}(k)\right\| & =\left\|\mathbb{E}\left\{\boldsymbol{z}(k) \boldsymbol{z}^{T}(k) 1_{\{\boldsymbol{\theta}(k)=i, \boldsymbol{\theta}(k+1)=j\}}\right\}\right\| \\
& \leq\left\|\mathbb{E}\left\{\boldsymbol{z}(k) \boldsymbol{z}^{T}(k)\right\}\right\|,
\end{aligned}
$$

it follows that $\lim _{k \rightarrow+\infty} \hat{Q}_{i, j}(k)=0$, and as the operator vec is continuous then $\lim _{k \rightarrow+\infty} \hat{q}_{i, j}(k)=0$. From here it concludes immediately (4.12).

Now, if $\lim _{k \rightarrow+\infty} \hat{q}(k)=0$, then $\lim _{k \rightarrow+\infty} \hat{q}_{i, j}(k)=0$ which is equivalent to saying that $\lim _{k \rightarrow+\infty} \hat{Q}_{i, j}(k)=0$. From (4.5) it is concluded that $\lim _{k \rightarrow \infty} \mathbb{E}\left\{\boldsymbol{z}(k) \boldsymbol{z}^{T}(k)\right\}=0$.

The following lemma gives us a connection between the systems 3.1 and 3.2.

Lemma 4.4. The solution $\boldsymbol{x}(k)$ of System (3.1) and the solution $\boldsymbol{z}(k)$ of System (3.2). The following is satisfied:

$$
\lim _{k \rightarrow \infty} \mathbb{E}\left\{\|\boldsymbol{x}(k)\|^{2}\right\}=0 \Leftrightarrow \lim _{k \rightarrow \infty} \mathbb{E}\left\{\|\boldsymbol{z}(k)\|^{2}\right\}=0
$$

Proof: The proof is essentially based on equality and inequality (3.3).

4.1. Test for MSS. The Theorem 4.1 provides an easy computational implementation tool to analyze if the System (3.1) is MSS using the spectral radius of the matrix $\mathcal{A}$.

Theorem 4.1. The System (3.1) is MSS if and only if $\rho(\mathcal{A})<1$.

Proof: If the System (3.1) is MSS, then by the inequality (4.6) is obtained $\lim _{k \rightarrow+\infty} \mathbb{E}\left\{\|\boldsymbol{x}(k)\|^{2}\right\}=0$, and by the Lemma 4.4 it follows $\lim _{k \rightarrow+\infty} \mathbb{E}\left\{\|\boldsymbol{z}(k)\|^{2}\right\}=0$, finally for the inequality (4.6) is obtained $\lim _{k \rightarrow+\infty} \mathbb{E}\left\{\boldsymbol{z}(k) \boldsymbol{z}^{T}(k)\right\}=0$ and by Lemma 4.3 it is concluded that $\lim _{k \rightarrow+\infty} \hat{q}(k)=0$. In addition, we have to $\boldsymbol{x}(0), \boldsymbol{x}(1)$ and $\boldsymbol{\theta}(0), \boldsymbol{\theta}(1)$ are arbitrary and independent considering that

$$
\begin{aligned}
\hat{Q}_{i, j}(0) & =\mathbb{E}\left\{\boldsymbol{z}(0) \boldsymbol{z}^{T}(0)\right\} \mathbb{E}\left\{1_{\{\boldsymbol{\theta}(0)=i\}} 1_{\{\boldsymbol{\theta}(1)=j\}}\right\} \\
& =\mathbb{E}\left\{\boldsymbol{z}(0) \boldsymbol{z}^{T}(0)\right\} p_{i, j} \pi_{i},
\end{aligned}
$$

then it is seen that it is always possible to obtain $\hat{q}(0)$ with non-zero components. Then by Jordan's decomposition of $\mathcal{A}$ and by (4.11) it follows that $\rho(\mathcal{A})<1$.

Now, if $\rho(\mathcal{A})<1$ then taking limit on both sides of (4.11) this implies that $\lim _{k \rightarrow+\infty} \hat{q}(k)=0$. From here, for the Lemma 4.3 and the inequality (4.6) it is concluded that the System (3.1) is MSS. This subsection shows an example that illustrates the results of Theorem 4.1.

Example 1. This example shows a system that is not MSS.

$$
A_{1}=B_{1}=\left[\begin{array}{ll}
3 & 0 \\
0 & 0
\end{array}\right] \quad A_{2}=B_{2}=\left[\begin{array}{ll}
0 & 0 \\
0 & 1
\end{array}\right], \quad \Pi=\left[\begin{array}{ll}
0 & 1 \\
1 & 0
\end{array}\right] .
$$

We noticed that $\rho(\mathcal{A})=3>1$, the system is not MSS. In fact, we have two significant accomplishments

$$
w_{1}=\{1,2,1,2, \cdots\} ; w_{2}=\{2,1,2,1, \cdots\} .
$$

For $w_{1}$, it has 


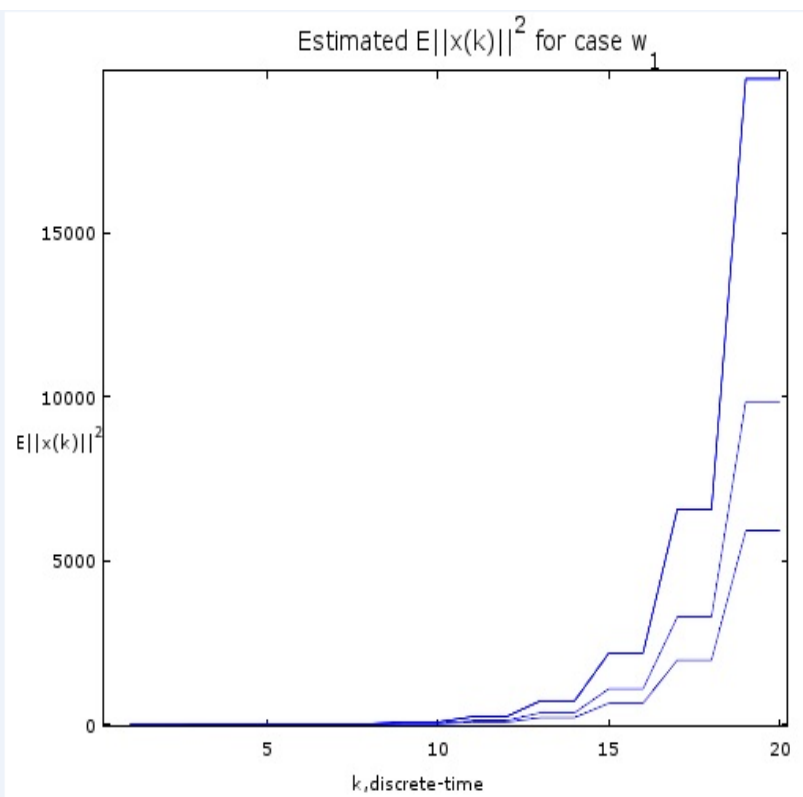

For $w_{2}$, it has

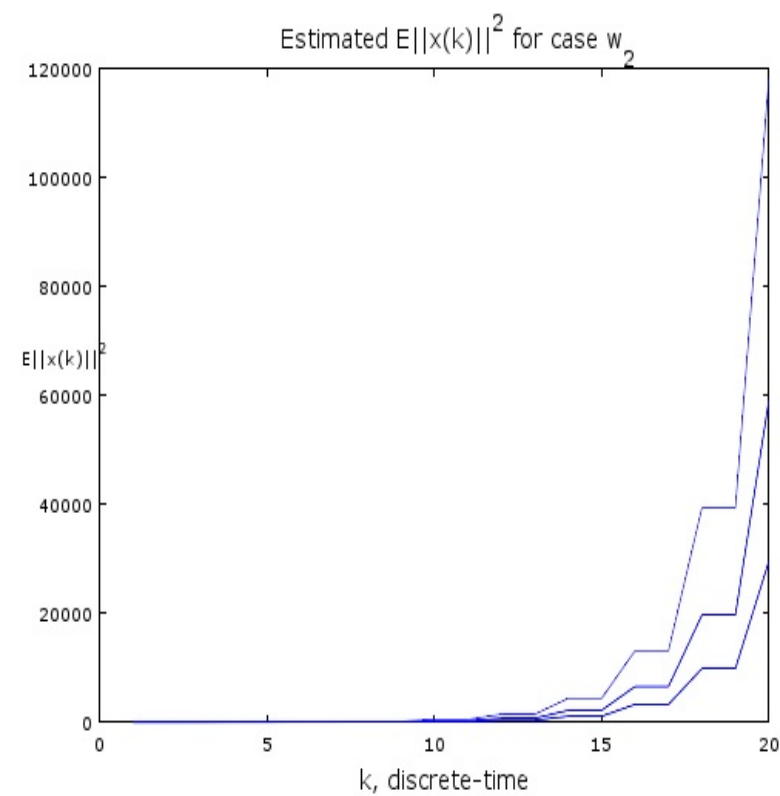

This implies that $\lim _{k \rightarrow+\infty} \mathbb{E}\left\{\|\boldsymbol{x}(k)\|^{2}\right\}=+\infty$.

5. Equivalence between stabilities. This section establishes the relationships between the different types of stability introduced in section 4 . It is proved that under the condition of being $\mathcal{L}$ a finite state space, the notions of stability $(a)-(c)$ are equivalent and all of them imply stability $(d)$.

We note that the system (3.2) is an MJLS, by classical theory it is known that the types of stability are equivalent. When you want to verify the same result for the system (3.1) there are technical difficulties you see the need to present some previous results presented below. The following theorem gives us an equivalence of stability MSS of System (3.1) and System (3.2).

Theorem 5.1. The System (3.1) is MSS if and only if the System (3.2) is MSS.

Proof: The proof is based of Lemma 4.4 and the inequality (4.6).

The following theorem gives us an equivalence of stability SS of System (3.1) and System (3.2).

Theorem 5.2. The System (3.1) is SS if and only if the System (3.2) is SS.

Proof: If the System (3.1) is SS and

$$
\sum_{k=0}^{\infty} \mathbb{E}\left\{\|\boldsymbol{z}(k)\|^{2}\right\}=\sum_{k=0}^{\infty} \mathbb{E}\left\{\|\boldsymbol{x}(k)\|^{2}\right\}+\sum_{k=0}^{\infty} \mathbb{E}\left\{\|\boldsymbol{x}(k+1)\|^{2}\right\}<\infty
$$

Conversely, if the System (3.2) is SS and $\|\boldsymbol{x}(k)\|^{2} \leq\|\boldsymbol{z}(k)\|^{2}$, it is concluded by the comparison criteria. We are ready to present the equivalence between MSS and MSES of the System (3.1). 
Theorem 5.3. The System (3.1) is MSS if and only if the System (3.1) is MSES.

Proof: If the System (3.1) is MSS then for the Theorem 4.1, implies that $\rho(\mathcal{A})<1$. We have to $\left\|\mathcal{A}^{k}\right\| \leq$ $\beta \gamma^{k}$ for some $1 \leq \beta$ and $\gamma \in\langle 0,1\rangle$ by similar arguments of Proposition 2.5 ( see [15]), it is concluded. Now, if the System (3.1) is MSES, it is trivially proof that the System (3.1) is MSS.

The following theorem gives us an equivalence of stability ASS of System (3.1) and System (3.2).

Theorem 5.4. If the System (3.1) is ASS, if and only if the System (3.2) is ASS.

Proof: The proof is essentially based on equality and inequality (3.3).

Remark Recall that for the System (3.2), MSS, SS and MSES stability are equivalent according to the theory of MJLS. For the results of Theorems [5.1-5.4] is obtained

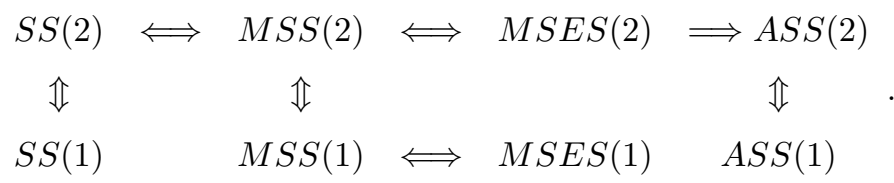

The last scheme indicates that the types of stability for the system (3.1) are equivalent and these imply the ASS.

6. Conclusions. In this paper, the three stability concepts for the System (3.1) have been introduced and proved to be equivalent under certain conditions. This is a new result and represents an extension of the well known result for the Markov linear jump system (MJLS) which sets the same property for the same three stability concept. A test is presented in terms of the spectral radius of a certain matrix to analyze the stability of the System (3.1) and then present an example and check the test.

7. Acknowledgements. The authors thank the authorities of the Faculty of Science of the National University of Engineering for their support and funding for such research. This research was supported by CAR-FC-UdI under grant 105-2018 and CAR-FC-UdI under grant 010-2020.

ORCID and License

Jorge Enrique Mayta Guillermo https: / / orcid.org/0000-0002-7872-1639

Maritza Lourdes Moreno Capristano https://orcid.org/0000-0002-7956-3775

This work is licensed under the Creative Commons Attribution-NoComercial-ShareAlike 4.0.

\section{References}

[1] Li X, Hui D, Lai X, Yan T. Power quality control in wind fuel cell battery hydrogen electrolyzer hybrid microgrid power system. In: Ognyan Ivanov editor. Applications and Experiences of Quality Control. Rijeka: INTECH; 2011. p. 579-594. Avalible form: https://www. intechopen. com /books/ applications-and-experiences-of-quality-control/power- quality- control-inwind-fuel-cell-battery-hydrogen-electrolyzer-hybrid-micro-grid-power-system

[2] Ugrinovskii V, Pota HR. Decentralized control of power systems via robust control of uncertain Markov jump parameter systems. Int. J. of Control. 2005; 78(9):662-677.

[3] Meskin N, Khorasani K. Fault detection and isolation of discrete-time Markovian jump linear systems with application to a network of multi-agent systems having imperfect communication channels. Automatica. 2009; 45(9):2032-2040.

[4] Gray WS, González OR, Dogan M. Stability analysis of digital linear flight controllers subject to electromagnetic disturbances. IEEE Trans. on Aerospace and Electronic systems. 2000; 36(4):1204-1218.

[5] Li J-R, Sculley J, Zhou, H-C. Metal-organic frameworks for separations. Chem. rev. 2012; 112(2):869-932.

[6] Sworder D, Rogers R. An LQ-solution to a control problem associated with a solar thermal central receiver. IEEE Trans. on Automatic Control. 1983; 28(10):971-978.

[7] Tesfaye S, Boulton AJ, Dyck PJ, Freeman R, Horowitz, M, Kempler P, Bernardi L. Diabetic neuropathies: update on definitions, diagnostic criteria, estimation of severity, and treatments. Diabetes care. 2010; 33(10):2285-2293.

[8] Blom HA, Bar-Shalom Y. The interacting multiple model algorithm for systems with Markovian switching coefficients. IEEE trans. on Automatic Control. 1988; 33(8):780-783.

[9] Brewer J. Kronecker products and matrix calculus in system theory. IEEE Trans. on circuits and systems. 1978; 25(9):772-781.

[10] Blankenship G. Stability of linear differential equations with random coefficients. IEEE Trans. on Automatic Control. 1977; 22(5):834-838.

[11] Feng X, Loparo KA, Ji Y, Chizeck HJ. Stochastic stability properties of jump linear systems. IEEE Trans. on Automatic Control. 1992; 37(1):38-53.

[12] Chizeck HJ, Willsky AS, Castanon D. Discrete-time Markovian-jump linear quadratic optimal control. Int. J. of Control. 1986; 43(1):213-231

[13] Ji Y, Chizeck HJ. Controllability, observability and discrete-time Markovian jump linear quadratic control. Int. J. of Control. 1988; 48(2):481-498.

[14] Fang Y, Loparo KA. Stochastic stability of jump linear systems. IEEE trans. on automatic control. 2002; 47(7):1204-1208.

[15] Costa OLV, Fragoso MD, Marques RP. Discrete-time Markov jump linear systems. New York: Springer Science \& Business Media; 2005. 